\title{
Preventive effects of intrauterine injection of bone marrow-derived mesenchymal stem cell- conditioned media on uterine fibrosis immediately after endometrial curettage in rabbit
}

\section{Sanaz Bazoobandi}

Shiraz University of Medical Sciences

Nader Tanideh

Shiraz University of Medical Sciences

Farhad Rahmanifar

Shiraz University

Shahrokh Zare

Shiraz University of Medical Sciences

Omid Koohi-Hosseinabadi

Shiraz University of Medical Sciences

Iman Razeghian Jahromi

Shiraz University of Medical Sciences

Mehdi Dianatpour

Shiraz University of Medical Sciences

\section{Zahra Khodabandeh}

Shiraz University of Medical Sciences

Masoumeh Ahmadi

Shiraz University of Medical Sciences

Arezoo Khoradmehr

Bushehr University of Medical Sciences

Iraj Nabipour

Bushehr University of Medical Sciences

Amin Tamadon ( $\nabla$ amintamaddon@yahoo.com )

Bushehr University of Medical Sciences https://orcid.org/0000-0002-0222-3035

\section{Research}

Keywords: Uterine fibrosis, Bone marrow, Mesenchymal Stem Cell, Conditioned medium

Posted Date: April 24th, 2020 
DOI: https://doi.org/10.21203/rs.3.rs-23917/v1

License: (c) (1) This work is licensed under a Creative Commons Attribution 4.0 International License. Read Full License 


\section{Abstract \\ Background}

Uterine fibrosis is a uterine acquired disorder with symptoms of implantation disturbances, menstrual irregularities and abortion. Mesenchymal stem cells (MSCs) have antifibrotic properties by secretion of chemokines. MSCs-conditioned media (MSC-CM) contains paracrine components, exosomes, with a great potential for repairing damaged tissue or prevention of fibrosis. The main goal of this study was to evaluate the preventive effects of bone marrow-derived MSC-CM (BM-MSC-CM) on uterine fibrosis after uterine curettage in rabbits.

\section{Methods}

This study included 12 female rabbits (24 uterine horns) that double uteri of each 12 female rabbits were randomly divided into four groups of intact negative control, curettage positive control, BM-MSCs injection, and BM-MSC-CM injection in the way that two corresponding uteri from a rabbit were assigned in different groups. The MSC-CM was collected from cultivated BM-MSCs $48 \mathrm{~h}$ after three times washing and replacement of serum free media. Through a surgical approach, except intact negative uteri, the caudal part of the uteri was submitted traumatic endometrial curettage. After suturing of uterine wall, BMMSC or BM-MSC-CM were injected in the curettage site. Endometrial regeneration was histologically evaluated 30 days after treatment.

\section{Results}

Curettage with or without preventive injections increased the growth of endometrial layer based on the histomorphometric indices evaluation. However, the amount of fibrotic tissue in the BM-MSC-CM injection group was lower than the BM-MSC and the curettage group, single injection of CM of MSCs after 30 days prevented the fibrotic effects of curettage on endometrial layer of rabbits.

\section{Conclusions}

Injecting BM-MSC-CM immediately after curettage prevented and reduced the uterine fibrosis better than BM-MSC in a rabbit model.

\section{Introduction}

Uterine fibrosis is caused by the destruction of the endometrium and a partial or complete closure of the uterus and leads to infertility, recurrent pregnancy losses, menstrual abnormalities after endometritis or aggressive curettages (1). Intrauterine adhesions can develop from lesion of the basal layer of the endometrium caused by curettage of the newly pregnant uterus. The syndrome may also occur after 
hysteroscopy surgery such as myomectomy, polypectomy and endometrial ablation, endometriosis, abortion, uterine artery embolization or uterine tuberculosis. Symptoms of uterine fibrosis include amenorrhea, abortion, and infertility. Several methods have been developed for treatment of uterine fibrosis. Surgical treatments using hysteroscopy to remove the adherent tissue inside the uterine cavity is the first option (2). Recently, Stem cell therapy (3) and stem cell-derived microvesicle injections $(4,5)$ have been recommended for treatment of uterine fibrosis. There is no modality to prevent uterine fibrosis after curettage

Stem cell therapy and its derivatives play an important role in medical and pharmaceutical sciences. Among many types of mesenchymal stem cells (MSCs), bone marrow-derived MSCs (BM-MSCs) are multipotent stem cells that are capable of differentiating into different somatic cells $(6,7)$. In recent years, the significant role of BM-MSCs is worthy of attention for treatment of uterine fibrosis. Stem cell therapy of uterine fibrosis using BM-MSC has been reported in animal models $(3,8)$ or human $(9)$. A clinical research also implicated that intrauterine transplantation of autologous BM-MSCs were beneficial for regeneration of the injured endometrium (10).

After transplantation, BM-MSCs can migrate and accumulate in endometrial tissue (3). It is commonly approved that transplanted cells can provide morphological and functional benefits through multiple mechanisms including, but not restricted to, trophic support, cell replacement, regeneration of endogenous cells, immunosuppression/anti-inflammation, stimulation, and regulatory interactions with endogenous cells (3). Hence, the BM-MSCs might have an important role in endometrial rebuilding, at least in terms of cellular exchanges and inflammatory stimuli (11). Stem cell therapy for regeneration of endometrium has proven to be a great promise for repair and regeneration of the injured tissues (12).

Since the preparation and administration of stem cells for treatment of patients with uterine fibrosis when uterine injuries occur simultaneously is not practical, the use of stem cells in the treatment of this disease is actually impaired. Therefore, providing an alternative approach instead of the direct use of stem cells byproducts is a necessity. Accumulative evidence suggests application of secreting microvisecles of MSCs rather than stem cells for treatment of uterine fibrosis $(4,5)$. Therefore, administration of the stem cell culture medium containing microvisecles for prevention of uterine fibrosis concurrent with uterine curettage may be a good alternative to stem cell therapy of this disease. This study used BM-MSCs conditioned media (CM) in a rabbit model of uterine fibrosis and compared it with BM-MSCs injection.

\section{Materials And Methods}

\section{Ethical approval statements}

This investigation was performed in accordance with relevant guidelines and regulations of animal studies and all experimental protocols were approved by ethical committee of Bushehr University of Medical Sciences (Permission number: IR.BPUMS.REC.1399.084).

\section{Animals}


Thirteen female ( 1 donor and 12 recipients) adult healthy New Zealand white albino rabbits (Oryctolagus cuniculus) weighing 3500-4000 g were purchased from and housed in the Center of Comparative and Experimental Medicine, Shiraz University of Medical Sciences. They were maintained singly in stainless steel cages under the appropriate condition $\left(20 \pm 2{ }^{\circ} \mathrm{C}\right.$ temperature, humidity $60 \%$, and 12 -h light/dark cycle) and had free access to food and water. Their food was supplemented by adding carrot and parsley.

\section{BM-MSCs isolation}

To establish the BM-MSCs culture, under sterile condition both femur and tibia from a rabbit were excised and carefully cleaned of adherent soft tissue. The ends of the bones were cut away and bone marrow was harvested by flushing with $10 \mathrm{~mL}$ syringe with Dulbecco Modified Eagle Medium (DMEM) supplemented with 10\% fetal bovine serum (FBS), 1\% penicillin and streptomycin (Sigma-Aldrich) and 1\% L-glutamine (Sigma-Aldrich). After washing and centrifugation at $1200 \mathrm{rpm}$ for $5 \mathrm{~min}$, cell pellet was resuspended in fresh medium and cultured in a $75-\mathrm{cm}^{2}$ flask in the supplemented DMEM medium. The flask was incubated in a $\mathrm{CO}_{2}$ incubator $\left(5 \% \mathrm{CO}_{2}, 37^{\circ} \mathrm{C}\right.$, and saturated humidity). The first culture media was changed after $24 \mathrm{~h}$ to remove non-adherent cells.

The adherent cells were cultured till $80-90 \%$ confluence by exchanging the subsequent medium every $3-$ 4 days and then passaged to expand the MSCs population. The adherent cells were washed with phosphate buffer saline (PBS) and the cells were harvested after for 2-3 min treating with $0.25 \%$ trypsin (Gibco) and the enzyme was inactivated with same amount of culture medium. Spindle-shaped morphology of BM-MSCs was observed using light microscopy at every passage. The cells were subcultured two times to obtain a sufficient number for evaluation of stemness characters and cell therapy. Cells in the second passage were collected and counted using a hemocytometer.

They were cryopreserved through the conventional method by dimethyl sulfoxide (DMSO; MP Bio, France) and were aliquoted into sterile cryovials at a density of $2 \times 10^{6}$ viable cells $/ \mathrm{ml}$. Before cell characterization or cell therapy, the frozen cryovials were quickly thawed in a $37^{\circ} \mathrm{C}$ water bath. Before the ice clump completely thawed, $1 \mathrm{~mL}$ of supplemented DMEM medium was added. After re-suspension of the cells in the fresh medium, they were cultured and subcultured just one time in the same condition and medium as has been explained above. Except cell morphology and plastic adherent characters of isolated cells, to confirm that the isolated cells were MSCs, the potential of differentiation to adipocytes and osteoblasts as well as their surface markers were detected.

\section{Reverse transcription-polymerase chain reaction (RT-PCR)}

BM-MSCs were examined for expression of surface markers using RT-PCR. Total RNA of BM-MSCs at passage 3 was extracted according to manufacturer's instructions using column RNA isolation kit (Denazist-Asia, Iran). Total RNA concentration was determined by nanodrop spectrophotometery. Before reverse transcription, the RNA samples were digested with DNase to remove contaminating genomic DNA. After that, complementary DNA (cDNA) synthesis from DNA-free RNA (500 ng) samples was done by using Accu Power Cycle Script RT PreMix Kit (Bioneer, Korea) according to the manufacturer's protocol. 
Specific primers were designed based on sequences corresponding to highly conserved regions of CD34, $\mathrm{CD} 45$, and CD73 in rabbit.The primer sequences used are summarized in Table 1. The micro-tubes containing requirements of PCR reaction up in a $20 \mu \mathrm{L}$ mixture were transferred to a Thermocycler (Eppendorf Mastercycler Gradient, Eppendorf, Hamburg, Germany). The RT-PCR amplification conditions for the surface markers were performed in 30 cycles of amplification including denaturation at $95^{\circ} \mathrm{C}$ for $30 \mathrm{sec}$, annealing at $64^{\circ} \mathrm{C}$ for $30 \mathrm{sec}$, and extension at $72{ }^{\circ} \mathrm{C}$ for $30 \mathrm{sec}$, with deployment 2 min at $95{ }^{\circ} \mathrm{C}$ for primary denaturation and $5 \mathrm{~min}$ at $72{ }^{\circ} \mathrm{C}$ for final extension. PCR products were run on $1.5 \%$ agarose gel electrophoresis and visualized by UV light (UVtec, Cambridge, UK).

Table 1

Sequences of RT-PCR primers used to quantify the expression of bone marrow-derived mesenchymal stem cells specific surface markers (CD73) and hematopoietic stem cells specific surface markers (CD45 and CD34) in rabbit

\begin{tabular}{|lll|}
\hline Primer & Primer sequence & Amplicon length (bp) \\
\hline CD34-F & ACCATCTCAGAGACTAGAGTC & 512 \\
CD34-R & GAAAGTTCTGTTCTGTTGGC & \\
CD45-F & CAGTACTCTGCCTCCCGTTG & 269 \\
CD45-R & TACTGCTGAGTGTCTGCGTG & \\
CD73-F & TACACCGGCAATCCACCTTC & 212 \\
CD73-R & CTTGGGTCTTCGGGAATGCT & \\
\hline
\end{tabular}

\section{Osteogenic and adipogenic differentiation assay}

In order to evaluate the differentiation potential of BM-MSCs, cells of passage 3 were used and osteogenic and adipogenic differentiation were induced. For osteogenic differentiation BM-MSCs were seeded in a 6-well plate. After the cells reaching 70\% confluence, they were cultured for 3 weeks in osteogenic medium containing low glucose DMEM supplement with $100 \mathrm{nM}$ dexamethasone (SigmaAldrich), $0.05 \mathrm{mM}$ ascorbate-2-phosphate (Wako Chemicals, Richmond, VA, USA), $10 \mathrm{mM}$ bglycerophosphate (Sigma-Aldrich), 1\% antibiotic/antimycotic and 10\% FBS. The half of medium was replaced every 3 days. At day 21, the cells were fixed by 10\% formalin solution (Sigma-Aldrich), and then stained using Alizarin red (Sigma-Aldrich) to detect calcified extracellular matrix and osteogenic differentiation.

For adipogenic differentiation BM-MSCs were seeded in a 6-well plate. When they reached $70 \%$ confluency, were induced to adipogenic differentiation with adipogenic induction medium containing DMEM low glucose, 10\% FBS, 0.5 mM isobutyl-methylxanthine (Sigma-Aldrich), 10\% FBS, 0.5 mM isobutyl-methylxanthine (Sigma-Aldrich), $1 \mu \mathrm{M}$ dexamethasone, $10 \mu \mathrm{M}$ insulin, $200 \mu \mathrm{M}$ indomethacin (Sigma-Aldrich). The plates were maintained for three weeks and medium was replaced every 3-4 days. 
At the end of period, the cultures were fixed by $10 \%$ formalin solution for 10 minutes. Fixed cells were subjected to oil red $\mathrm{O}$ (Sigma-Aldrich), which specifically stains lipid droplets.

\section{Cell counting, growth curve and calculation of population doubling time (PDT)}

Growth curves were plotted for BM-MSCs derived from rabbit bone marrow in order to evaluate growth kinetics of the cells. For the assessment of growth characteristics, BM-MSCs at passage 3 were seeded in a 24-well plate at a density of approximately $7.5 \times 10^{4}$ cells per well in triplicate. Cells were collected from each well 1-7 days after seeding and counted microscopically to draw a cell growth curve. The curve was drawn using GraphPad Prism (Version 5.01; GraphPad software Inc., San Diego, CA, USA).

To evaluate the in-vitro proliferation rate, the PDT value was determined for each studied cells. PDT was calculated using the formula PDT $=\mathrm{T} \ln 2 / \mathrm{In}(\mathrm{Xe} / \mathrm{Xb})$, in which $\mathrm{T}$ is the incubation time in hours, $\mathrm{Xb}$ represents the cell number at the beginning of the incubation time and Xe corresponds to the cell number at the end of incubation time.

\section{Preparation of BM-MSC-CM}

The MSC-CM was collected from the third passages of cultivated BM-MSCs. In order to obtain the CM, BM-MSCs at passage three were cultured at a density of $10^{6}$ cells per T75 flask. At 80 to $90 \%$ confluence, the BM-MSCs were washed three times with PBS and the media were replaced with $10 \mathrm{~mL}$ of FBS-free DMEM. After $48 \mathrm{~h}$ incubation, the media were collected and filtrated through a $0.2-\mu \mathrm{m}$ filter to remove cellular debris and stored at $-80{ }^{\circ} \mathrm{C}$ until use.

\section{Surgical procedure}

For induction of the model and treatment, double uteri of each 12 female rabbits were randomly divided into four groups of intact negative control, curettage positive control, BM-MSCs injection, and BM-MSC$\mathrm{CM}$ injection in the way that two corresponding uteri from a rabbit were assigned in the different groups $(n=6)$. Except intact negative uteri, the caudal part of the other uteri was submitted traumatic endometrial curettage.

Briefly, the rabbits were anesthetized with a single intramuscular dose of a combination of ketamine $10 \%$ (35-40 mg/kg, Alfasan, Netherlands), and xylazine 2\% (3-5 mg/kg, Alfasan, Netherlands) after preoperative overnight fasting. Following midline incision as laparotomy, $5 \mathrm{~cm}$ long incisions were performed on the uteri. Incisions were located in the middle of each uterine tube. Using a scalpel blade through these incisions, the inside out everted endometrium was scratched (Fig. 1A). Whole thickness of endometrium was removed and it was continued until observation of bleeding as an indicator of curettage completion. Then uteri wall was sutured with 4-0 vicryl. The curettage positive control uteri were curettaged but not injected. Immediately, after suturing of uterus in both treatment groups, $1 \mathrm{~mL}$ of the BM-MSCs $\left(2 \times 10^{6}\right.$ cells) or BM-MSC-CM were injected into the uterus (Fig. 1B). The intact negative control uteri were not curettaged or injected. Using 3 - 0 vicryl, abdominal muscles were sutured and by 2 
- 0 silk sutures, the skin was closed. At the end of operation, rabbits received flunixin meglumine $\left(0.2 \mathrm{mg} / \mathrm{kg}, \mathrm{IM}\right.$, Caspian-Tamin ${ }^{\mathrm{T}}$, Iran) immediately and then every 24 hours in 3 doses. Penstrep-400 (Nasr ${ }^{\text {TM }}$, Iran, IM) was injected as an antimicrobial, just after the operation and continued for 3 days.

\section{Histomorphometry of uterus}

Effect of both treatment techniques on regeneration of endometrium evaluated 30 days after treatment by histomorphometric comparing with the controls. Rabbits were sacrificed with a high dose of pentobarbitone (1 g, Specia, France). Both uteri of rabbits were fixed for two weeks in $10 \%$ formalin buffer. After fixation, segments were embedded in paraffin, and $5 \mu \mathrm{m}$ thickness sections were made from each block in the area of curettage with the help of suture landmark.

They were stained with Masson trichrome to analyze histopathologic damages following curettage and the evolution of the regeneration process after treatment by evaluating morphologic indices and accumulation of fibrotic tissues in the curettage site. The slides were visualized and photographed using a light microscope (CX21, Olympus, Japan) equipped with an adjusted digital camera (AM423U Eyepiece Camera, Dino-Eye, Taiwan). On the transverse sections, endometrial area, lumen area, and total area of the uterus were measured using Dinocapture 2.0 software (Dino-Eye, Taiwan). The proportion of intact, damaged and regenerated endometrial luminal epithelium were estimated by calculation of different indices. Histomorphometric indices according to the previously described method (13) include lumen area/total horn area ratio (LA/THA), endometrial area/total horn area ratio (EA/THA), myometrial and perimetrial area/total horn area ratio (MPA/THA), endometrial area/uterine wall area ratio (EA/UWA), and myometrial and perimetrial area/uterine wall area ratio (MPA/UWA). Synechiae occurring, inflammatory elements and histopathologic changes were evaluated in intact, curettage and treatment uteri.

\section{Image analysis of fibrosis}

Three separate fields $\left(200 \times 200 \mu \mathrm{m}^{2}\right)$ of endometrial layer in 1 slides $(n=6)$ from different groups were cropped. Then, using color threshold of ImageJ, the green and blue pixels which showed the presence of collagen fibers were selected and percent of area of collagen were measured in each field.

\section{Statistical analysis}

Normality test of the histomorphometric ratio indices were carried out by Kolmogorov-Smirnov test. The mean and standard error (SE) ratios of LA/THA, EA/THA, MPA/THA, EA/UWA and MPA/THA were subjected to Kolmogorov-Smirnov normal test and data were analyzed by two-way ANOVA and LSD tests. Data were analyzed by SPSS 22 for windows (IBM SPSS Statistics for Windows, version 22, IBM Inc., Chicago, Illinois). P-value less than 0.05 was considered statistically significant. Means and SE are reported in charts (GraphPad Prism version 5.01 for Windows, GraphPad Inc. Inc., San Diego, CA, USA).

\section{Results}

\section{Rabbit BM-MSCs culture}


In the primary culture, the cells had various forms; however, the spindle cells or fibroblast-like cells which are naturally occurring forms of MSCs had the highest number among others; although flat or rounded cells were visible, too. Over time, the cells were multiplied and gradually became denser. Thus, the cell colonies were detectable in culture plate, these cell aggregations, in turn, attached to each other with the growth of cells and consequently, the whole of culture medium was full of cells after about two weeks. When the culture medium was filled of BM-MSCs, the cells were passaged using trypsin/EDTA. Rate of cell growth and proliferation were increased from this passage which was called passage one to next passages, such that filled out cultivation surface in about three to four days. It should be noted that at this stage, the cells are often seen in the form of fibroblast-like cells or spindle cells (Fig. 2A). This process continued until the third passage.

\section{Rabbit BM-MSCs characterization}

To further confirmation of the MSCs characters in isolated cells, the osteogenic and adipogenic differentiation capacity of BM-MSCs were evaluated. After culture of BM-MSCs in osteogenic and adipogenic differentiation media separately, the cells were differentiated toward osteoblasts and also showed the presence of intracellular lipid droplets of adipocytes as respectively verified by positive staining with Alizarin red staining (Fig. 2B) and oil red $\mathrm{O}$ staining (Fig. 2C). To approve the expression of surface marker of MSCs of rabbit on isolated BM-MSCs, the cells were analyzed using a RT-PCR assay. Figure 2D displays positive expression for MSCs marker (CD73) and negative expression for hematopoietic stem cell markers (CD34 and CD45). According to our result, the PDT of the passages 3 of the BM-MSCs were $53.7 \mathrm{~h}$ (Fig. 3). BM-MSCs showed a good proliferation rates in passage 3 . In this study the proliferation rate of BM-MSCs decreased gradually after 6 days.

\section{Histopathologic evaluation of uterine fibrosis prevention}

The normal morphology of uteri was demonstrated in the intact group (Fig. 4A). In curettage group, uteri were with moderate abnormal morphology and thinner endometrium which were referred as model group. In all curettage uteri, the histologic evidence of fibrosis was confirmed by Masson trichrome staining (Fig. 4B). Although the mean of percent of fibrosis tissue in BM-MSC-CM group (Fig. 4C) was lower than the curettage and BM-MSC groups (Fig. 4D), but no significant difference in the percent of fibrosis was observed between the curettage and treated groups (Fig. 5). The confirmed fibrosis was about $25 \%$ of endometrial layer in the curettage group (Fig. 5). There was no significant difference in percent of fibrosis between BM-treatment groups and the control group (Fig. 5). The means of percent of fibrosis tissue of BM-MSC-CM group and control group were closer than BM-MSC group.

\section{Histomorphometric indices}

Evaluations of histomorphometric indices including the LA/THA ratio, EA/THA ratio, MPA/THA ratio showed that growth of endometrial layers in the curettage and the treated groups had growth more than control group after 30 days $(P<0.05$; Figs. 6$)$. Curettage, BM-MSC-CM treatment and BM-MSC treatment 
groups had significant lower means of the lumen area/total area ratio than the intact control group $(\mathrm{P}<$ 0.05; Figs. 6A). Moreover, curettage, BM-MSC-CM treatment and BM-MSC treatment groups had significant higher means of the EA/THA ratio than the intact control group $(P<0.05$; Figs. 6B). Compare the mean of the MPA/THA ratio showed that there were no significant differences between all four groups ( $P>0.05$; Figs. 6C).

Evaluations of histomorphometric indices including the EA/UWA ratio and MPA/UWA ratio showed that growth of endometrial layers in the curettage and the treated groups had growth more than control group after 30 days $(\mathrm{P}<0.05$; Figs. 7). Curettage, BM-MSC-CM treatment and BM-MSC treatment groups had significant higher means of the endometrial area/uterine wall area ratio than the intact control group $(\mathrm{P}<$ 0.05; Figs. 7A). Moreover, curettage, BM-MSC-CM treatment and BM-MSC treatment groups had significant higher means of the myometrial + perimetrial area/uterine wall area ratio than the intact control group $(P<0.05$; Figs. 6B).

\section{Discussion}

Injection of BM-MSC-CM immediately after curettage reduced formation of fibrotic tissues in rabbit uterus but did not have any effect on uterine wall thickness. In addition, injection of BM-MSC-CM had slightly higher preventive effect than BM-MSC injection on formation of fibrotic tissues. Consistent with our findings, Liu, Hu (5) and Ho, Lan (4) using a full uterine punch model showed the therapeutic effect of the MSC-CM for uterine fibrosis. CM contains exosomes is secreted from the cells (14) and could induce tissue regeneration $(15,16)$ or cell differentiation $(17,18)$. Bone marrow derived- $(19)$, uterine derived- $(20)$ and adipose derived- (21) MSCs exosomes also could restore endometrial function in a rat model of adhesions. These exosomes contains miRNAs which induce tissue regeneration (22). In a study by Xiao, Zhu (23), a positive effect of miR-340 on the function of cell therapy of Asherman's syndrome was confirmed. Furthermore, comparing BM-MSC-CM and MSC-CM regarding the time of regeneration, consistent with our findings, MSCs exosomal treatment restored the damage of Asherman's syndrome at tissue at a shorter time than the MSCs (20). Therefore, injecting BM-MSC-CM can be used for prevention of uterine fibrosis if it immediately injected after curettage.

Allotransplantation of BM-MSC immediately after curettage reduced formation of fibrotic tissues in rabbit uterus but did not have effect on uterine wall thickness. Before the studies showed the importance of BMMSCs in induction of endometrial regeneration, preliminary studies showed BM-derived cells (BMDCs) can differentiate into endometrial cells. BMDCs injection in uteri of the cellular proliferation-suppressed mice resulted in an increase in endometrial thickness $(24,25)$. Furthermore, allotransplantation of BMDCs from male to female uterus can produce endometrial tissue (26). As the BM contains both hematopoietic and mesenchymal cells, two groups of studies showed the effect of hematopoietic stem cells (HSCs) and MSCs on induction of uterine regeneration. HSCs injection could induce an increase in the thickness of a thin endometrium or formation of endometrial blood vessels (27-29). Beyond animal models, BMDCs and HSCs also treated Asherman's syndrome in human $(10,30,31)$. 
On the other hand, because uterine epithelium tissue conations endometrial MSCs (32-35), therefore the role of transplanted MSCs in prevention of uterine tissue fibrosis looks logic. But, during uterine injury, endometrial MSCs and their microenvironments disturbed and the potential of uterus for tissue regeneration decrease. Therefore, fibrotic tissue as a pathological healing process replaces normal endometrial tissue. In the current study, BM-MSCs injected immediately after curettage to prevent fibrosis tissue formation. To the best of our knowledge this method of stem cell therapy for prevention of fibrosis has not investigated. However, in previous studies the treatment of uterine injury after fibrosis formation showed that, MSCs injection improved regeneration of thin endometrium in mouse $(36,37)$ and rat $(38$, 39). For endometrial regeneration, various sources of MSCs have been used including allotransplantation of BM-MSCs (12), endometrial MSCs (37) and adipose MSCs $(21,39)$ or xenotransplantation of human amniotic MSCs (40). Therefore, findings of our study in combination with previous findings of uterine fibrosis treatment confirmed the preventive effects of BM-MSCs on uterine fibrosis.

In our study, for the first time rabbit model of uterine fibrosis has been used for treatment of uterine fibrosis which was induced by uterine curettage. The methods of induction of uterine damage are different including mechanical (36) or chemical (37) damages. Mechanical methods such using punch of uterus $(4,5)$ is not similar to human curettage of endometrium. However, various species of fibrosis induction in uterus have been used in different studies for MSC therapy including mice (36) and rat (38, 39), but rabbit may have some more benefit than them. In the present study, we used rabbit model due to its larger size of uterus than murine models. Larger size of uterus prepare a condition for controllable investigation of endometrial damage. Furthermore, the timing of estrous cycles in murine is 4 to 5 days and hormonal alterations of low duration estrus cycle may affect the similarity of these models to human uterine tissue regeneration. While, rabbit has longer diestrus phase than murine and uterine fibrosis model of rabbit $(13,41)$ look likes more similar to human than murine models.

Considering the fact that development of an applied method for prevention of uterine fibrosis using cell therapy methods need a protocol which can be done in emergency condition, method of intrauterine exosome injection as an expensive protocol or CM injection as a cheap solution immediately after curettage can be suggested. Allotransplantation injection of CM may permit the operator to save the therapeutic in standard condition and use it in the appropriate time. However, human clinical trials are necessary to confirm it.

\section{Conclusions}

Based on the findings of the current study, injecting BM-MSC-CM immediately after curettage have more effective role in preventing and reducing the uterine fibrosis than BM-MSC injection in a rabbit model. Therefore, the stressful condition of cell therapy and its possible complications can be removed by BMMSC-CM injection method for prevention of uterine fibrosis.

\section{List Of Abbreviations}


BMDCs, bone marrow-derived cells; BM-MSCs, bone marrow-derived mesenchymal stem cells; cDNA, complementary DNA; CM, conditioned media; DMEM, Dulbecco modified Eagle medium; DMSO, dimethyl sulfoxide; EA/THA, endometrial area/total horn area ratio; EA/UWA, endometrial area/uterine wall area ratio; FBS, fetal bovine serum; HSCs, hematopoietic stem cells; LA/THA, lumen area/total horn area ratio; MPA/THA, myometrial and perimetrial area/total horn area ratio; MPA/UWA, myometrial and perimetrial area/uterine wall area ratio; MSCs, mesenchymal stem cells; PBS, phosphate buffer saline; PDT, population doubling time; RT-PCR, reverse transcription-polymerase chain reaction

\section{Declarations}

\section{Ethics approval and consent to participate}

This investigation was performed in accordance with relevant guidelines and regulations of animal studies and all experimental protocols were approved by ethical committee of Bushehr University of Medical Sciences (Permission number: IR.BPUMS.REC.1399.084).

\section{Consent for publication}

Not applicable.

\section{Availability of data and materials}

The data used to support the findings of this study are available from the corresponding author upon request.

\section{Competing interests}

There is no conflict of interest.

\section{Funding}

This work was supported by Vice-Chancellor of Research and Technology, Bushehr University of Medical Sciences; Grant no. 1454.

\section{Authors' contributions}

S.B., A.T., N.T., F.R., H.N., S.Z., O.K.H., I.R.J., Z.K., M.D., M.A., A.K. and I.N. performed the experiments, analyzed the data, and co-wrote the draft. A.T., N.T., and F.R., designed the experiments, supervised the research, and co-wrote the paper. 


\section{Acknowledgements}

Not applicable.

\section{References}

1. Asherman JG. Traumatic intrauterine adhesions and their effects on fertility. Int J Fertil. 1957;2(1):49-61.

2. Dreisler E, Kjer JJ. Asherman's syndrome: current perspectives on diagnosis and management. Int J Womens Health. 2019;11:191-8.

3. Cervelló I, Gil-Sanchis C, Santamaría X, Cabanillas S, Díaz A, Faus A, et al. Human CD133 + bone marrow-derived stem cells promote endometrial proliferation in a murine model of Asherman syndrome. Fertil Steril. 2015;104(6):1552-60. e3.

4. Ho C-H, Lan C-W, Liao C-Y, Hung S-C, Li H-Y, Sung Y-J. Mesenchymal stem cells and their conditioned medium can enhance the repair of uterine defects in a rat model. J Chin Med Assoc. 2018;81(3):268-76.

5. Liu F, Hu S, Yang H, Li Z, Huang K, Su T, et al. Hyaluronic acid hydrogel integrated with mesenchymal stem cell-secretome to treat endometrial injury in a rat model of Asherman's syndrome. Adv Healthc Mater. 2019;8(14):e1900411.

6. Katagiri H, Kushida Y, Nojima M, Kuroda Y, Wakao S, Ishida K, et al. A distinct subpopulation of bone marrow mesenchymal stem cells, muse cells, directly commit to the replacement of liver components. Am J Transplant. 2016;16(2):468-83.

7. Salem M, Mirzapour T, Bayrami A, Sagha M. Germ cell differentiation of bone marrow mesenchymal stem cells. Andrologia. 2019;51(4):e13229.

8. Aghajanova L, Horcajadas JA, Esteban FJ, Giudice LC. The bone marrow-derived human mesenchymal stem cell: potential progenitor of the endometrial stromal fibroblast. Biol Reprod. 2010;82(6):1076-87.

9. Cervello I, Gil-Sanchis C, Mas A, Faus A, Sanz J, Moscardo F, et al. Bone marrow-derived cells from male donors do not contribute to the endometrial side population of the recipient. PLoS ONE. 2012;7(1):e30260.

10. Santamaria X, Cabanillas S, Cervello I, Arbona C, Raga F, Ferro J, et al. Autologous cell therapy with CD133 + bone marrow-derived stem cells for refractory Asherman's syndrome and endometrial atrophy: a pilot cohort study. Hum Reprod. 2016;31(5):1087-96.

11. Gargett CE, Nguyen HP, Ye L. Endometrial regeneration and endometrial stem/progenitor cells. Rev Endocr Metab Disord. 2012;13(4):235-51.

12. Gao L, Huang Z, Lin H, Tian Y, Li P, Lin S. Bone marrow mesenchymal stem cells (BMSCs) restore functional endometrium in the rat model for severe Asherman syndrome. Reprod Sci. 2019;26(3):436-44. 
13. Bazoobandi S, Tanideh N, Rahmanifar F, Tamadon A, Keshtkar M, Mehrabani D, et al. Induction of Asherman's syndrome in rabbit. J Reprod Infertil. 2016;17(1):10-6.

14. Park K-S, Bandeira E, Shelke GV, Lässer C, Lötvall J. Enhancement of therapeutic potential of mesenchymal stem cell-derived extracellular vesicles. Stem Cell Res Ther. 2019;10(1):288.

15. Almeria C, Weiss R, Roy M, Tripisciano C, Kasper C, Weber V, et al. Hypoxia conditioned mesenchymal stem cell-derived extracellular vesicles induce increased vascular tube formation in vitro. Front Bioeng Biotechnol. 2019;7:292.

16. Mehrabani D. Healing effect of conditioned media from bone marrow-derived stem cells in thioacetamide-induced liver fibrosis of rat. J Med Sci. 2016;16(1-2):7-15.

17. Razeghian Jahromi I, Mehrabani D, Mohammadi A, Ghahremani Seno MM, Dianatpour M, Zare S, et al. Emergence of signs of neural cells after exposure of bone marrow-derived mesenchymal stem cells to fetal brain extract. Iran J Basic Med Sci. 2017;20:301-7.

18. Lacham-Kaplan $\mathrm{O}$, Chy $\mathrm{H}$, Trounson A. Testicular cell conditioned medium supports differentiation of embryonic stem cells into ovarian structures containing oocytes. Stem Cells. 2006;24(2):266-73.

19. Yao Y, Chen R, Wang G, Zhang Y, Liu F. Exosomes derived from mesenchymal stem cells reverse EMT via TGF- $\beta 1 /$ Smad pathway and promote repair of damaged endometrium. Stem Cell Res Ther. 2019;10(1):225-.

20. Saribas GS, Ozogul C, Tiryaki M, Pinarli FA, Kilic SH. Effects of uterus derived mesenchymal stem cells and their exosomes on asherman's syndrome. Acta Histochem. 2020;122(1):151465.

21. Zhao S, Qi W, Zheng J, Tian Y, Qi X, Kong D, et al. Exosomes Derived from Adipose Mesenchymal Stem Cells Restore Functional Endometrium in a Rat Model of Intrauterine Adhesions. Reprod Sci. 2020:1-10.

22. Gong M, Yu B, Wang J, Wang Y, Liu M, Paul C, et al. Mesenchymal stem cells release exosomes that transfer miRNAs to endothelial cells and promote angiogenesis. Oncotarget. 2017;8(28):45200.

23. Xiao B, Zhu Y, Huang J, Wang T, Wang F, Sun S. Exosomal transfer of bone marrow mesenchymal stem cell-derived miR-340 attenuates endometrial fibrosis. Biol Open. 2019;8(5):bio039958.

24. Du H, Taylor HS. Contribution of bone marrow-derived stem cells to endometrium and endometriosis. Stem Cells. 2007;25(8):2082-6.

25. Du H, Naqvi H, Taylor HS. Ischemia/reperfusion injury promotes and granulocyte-colony stimulating factor inhibits migration of bone marrow-derived stem cells to endometrium. Stem Cells Dev. 2012;21(18):3324-31.

26. Ikoma T, Kyo S, Maida Y, Ozaki S, Takakura M. Bone marrow-derived cells from male donors can compose endometrial glands in female transplant recipients. Am J Obstet Gynecol. 2009;201(608):e1-8.

27. Bratincsák A, Brownstein MJ, Cassiani-Ingoni R, Pastorino S, Szalayova I, Tóth ZE, et al. CD45positive blood cells give rise to uterine epithelial cells in mice. Stem Cells. 2007;25(11):2820-6. 
28. Mints M, Jansson M, Sadeghi B, Westgren M, Uzunel M. Endometrial endothelial cells are derived from donor stem cells in a bone marrow transplant recipient. Hum Reprod. 2008;23:139-43.

29. Alawadhi F, Du H, Cakmak H, Taylor HS. Bone marrow-derived stem cell (BMDSC) transplantation improves fertility in a murine model of Asherman's syndrome. PLoS ONE. 2014;9(5):e96662.

30. Nagori CB, Panchal SY, Patel $\mathrm{H}$. Endometrial regeneration using autologous adult stem cells followed by conception by in vitro fertilization in a patient of severe Asherman's syndrome. J Hum Reprod Sci. 2011;4(1):43.

31. Singh N, Mohanty S, Seth T, Shankar M, Bhaskaran S, Dharmendra S. Autologous stem cell transplantation in refractory Asherman's syndrome: a novel cell based therapy. J Hum Reprod Sci. 2014;7(2):93.

32. Gargett CE, Schwab KE, Zillwood RM, Nguyen HP, Wu D. Isolation and culture of epithelial progenitors and mesenchymal stem cells from human endometrium. Biol Reprod. 2009;80(6):1136-45.

33. Tamadon A, Mehrabani D, Zarezadeh Y, Rahmanifar F, Dianatpour M, Zare S. Caprine endometrial mesenchymal stromal stem cell: multi-lineage potential, characterization and growth kinetics in breeding and anestrous stages. Vet Med Int. 2017;2017:5052801.

34. Mehrabani D, Rahmanifar F, Mellinejad M, Tamadon A, Dianatpour M, Zare S, et al. Isolation, culture, characterization, and adipogenic differentiation of heifer endometrial mesenchymal stem cells. Comp Clin Pathol. 2015;24(5):1159-64.

35. Ghobadi F, Rahmanifar F, Mehrabani D, Tamadon A, Dianatpour M, Zare S, et al. Endometrial mesenchymal stem stromal cells in mature and immature sheep: an in vitro functional study. Int $\mathrm{J}$ Reprod Biomed. 2018;16(2):83-92.

36. Li B, Zhang Q, Sun J, Lai D. Human amniotic epithelial cells improve fertility in an intrauterine adhesion mouse model. Stem Cell Res Ther. 2019;10(1):257-.

37. Kim YY, Park K-H, Kim YJ, Kim MS, Liu HC, Rosenwaks Z, et al. Synergistic regenerative effects of functionalized endometrial stromal cells with hyaluronic acid hydrogel in a murine model of uterine damage. Acta Biomater. 2019;89:139-51.

38. Jing Z, Qiong Z, Yonggang W, Yanping L. Rat bone marrow mesenchymal stem cells improve regeneration of thin endometrium in rat. Fertil Steril. 2014;101(2):587-94. e3.

39. Kilic S, Yuksel B, Pinarli F, Albayrak A, Boztok B, Delibasi T. Effect of stem cell application on Asherman syndrome, an experimental rat model. J Assist Reprod Genet. 2014;31(8):975-82.

40. Gan L, Duan H, Xu Q, Tang Y-Q, Li J-J, Sun F-Q, et al. Human amniotic mesenchymal stromal cell transplantation improves endometrial regeneration in rodent models of intrauterine adhesions. Cytotherapy. 2017;19(5):603-16.

41. Khrouf M, Morel O, Hafiz A, Chavatte-Palmer P, Fernandez H. Evaluation of the rabbit as an experimental model for human uterine synechia. J Hum Reprod Sci. 2012;5(2):175-80.

\section{Figures}




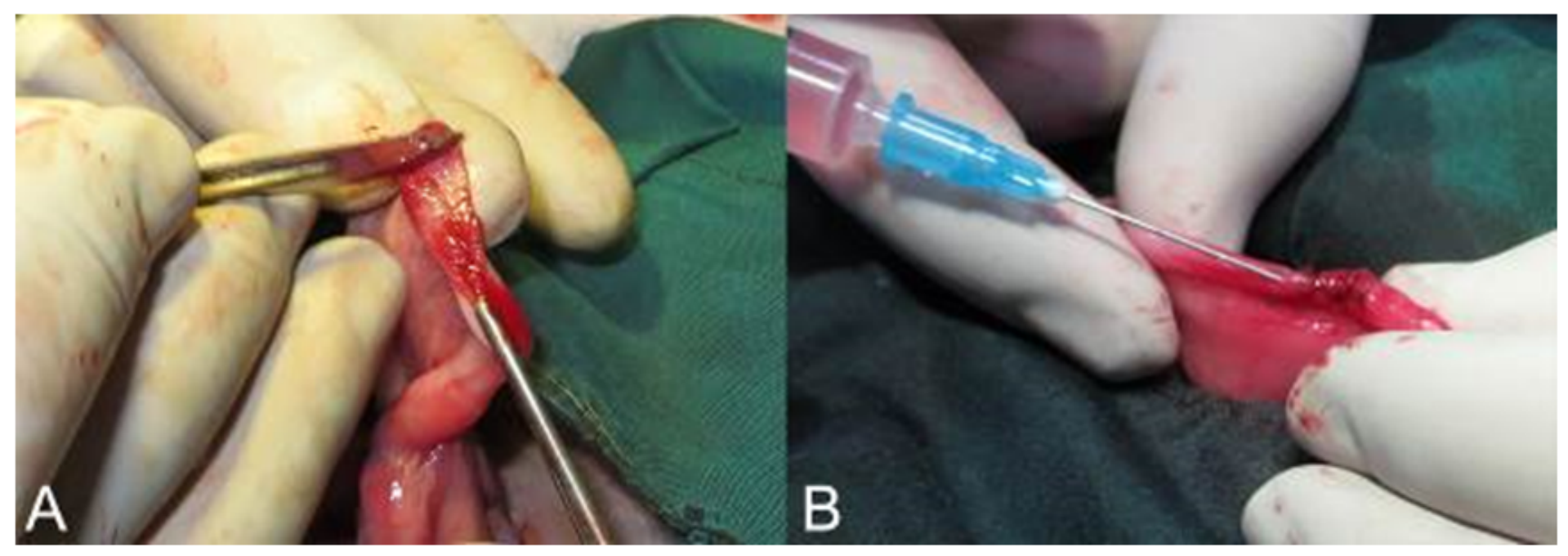

\section{Figure 1}

Surgical procedure of the evaluation of preventive effects of intrauterine stem cell-conditioned media injection on uterine fibrosis after endometrial curettage in rabbit $A$, intrauterine curettage of the rabbit uterus using a scalpel blade. $B$, injection of stem cells or stem cell-conditioned media after suturing of curettage uterus in treatment groups

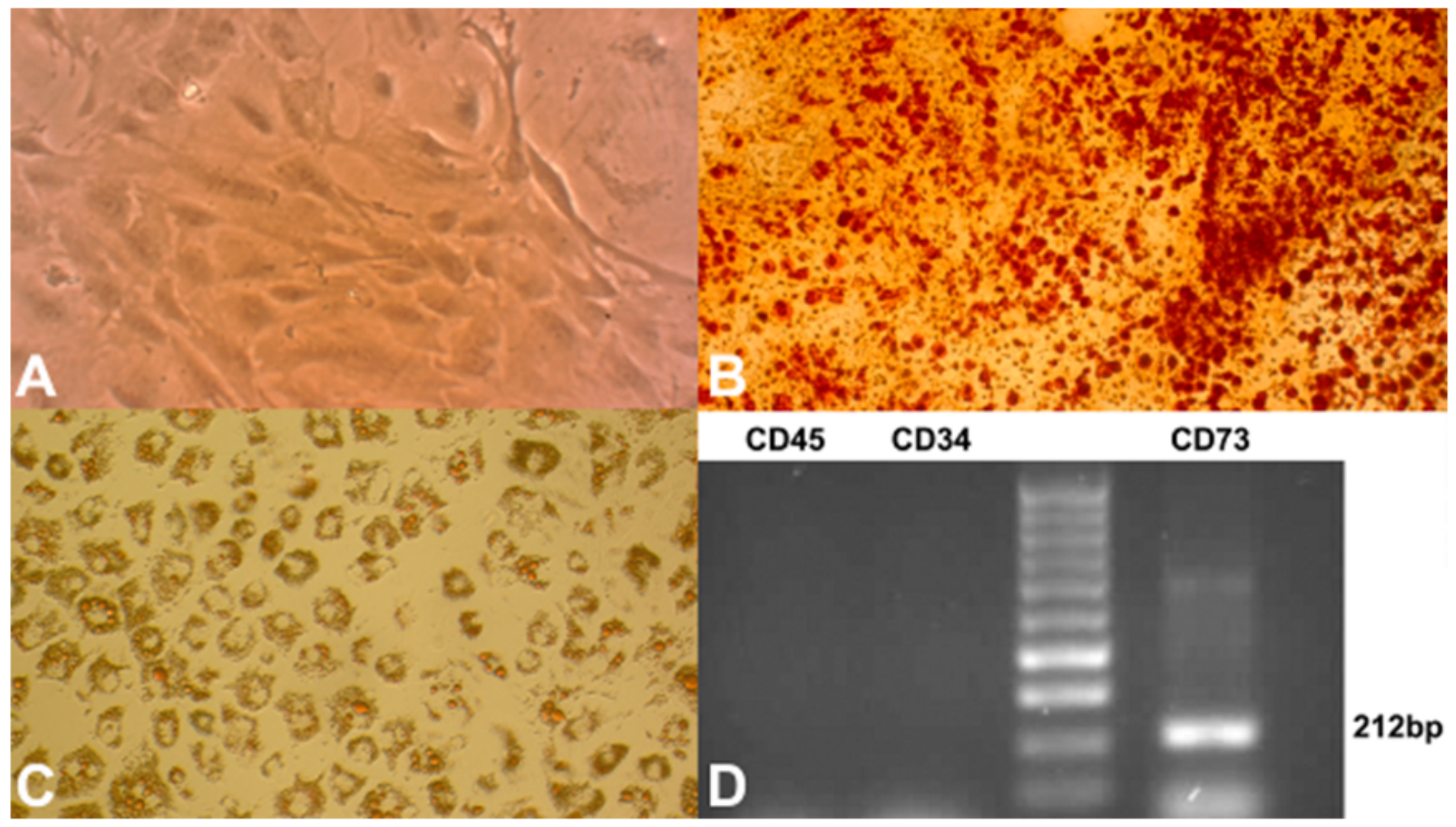

Figure 2 
Characterization of rabbit bone marrow-derived mesenchymal stem cells (BM-MSCs). A) Fibroblast-like and spindle shape morphology of rabbit BM-MSCs a typical characteristic of MSCs in passage $3,(\times 400)$. B) BM-MSCs of rabbit cultivated in osteogenic medium and stained with Alizarin red $(\times 100)$. C) BM-MSCs of rabbit cultivated in adipogenic medium and were stained with oil red $O$ at day 21 after induction $(\times 200)$. D) Agarose gel electrophoresis of products of reverse transcriptase polymerase chain reaction (RT-PCR) of BM-MSCs revealing to be positive for CD73 (mesenchymal marker) and negative for CD34 and CD45 (hematopoietic markers).

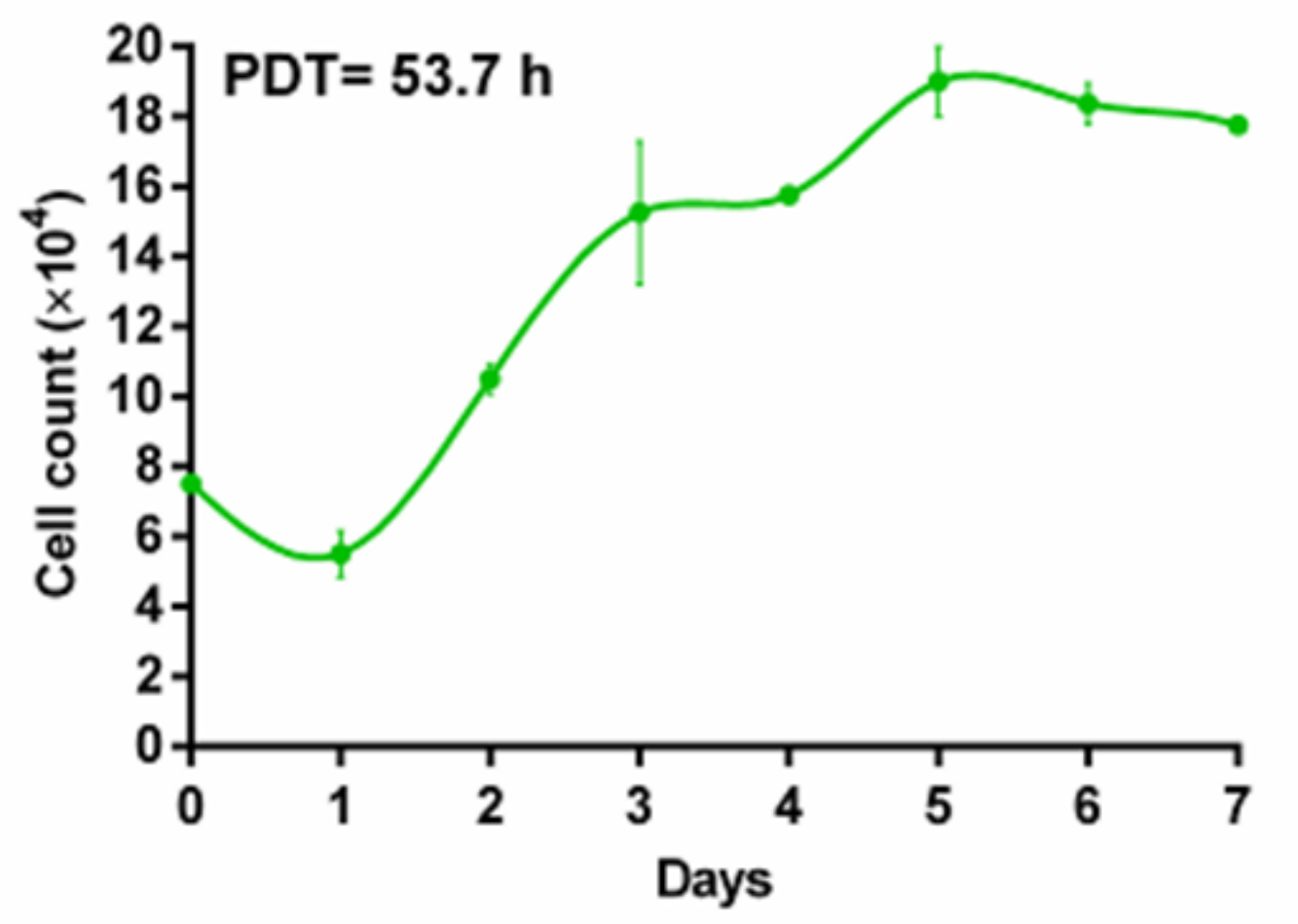

Figure 3

Mean and standard error of cell counts in growth curves of rabbit bone marrow-derived mesenchymal stem cells, passage 3. PDT, population doubling time.

\section{Figure 4}

Histological evaluation of the preventive effects of intrauterine bone marrow-derived stem cell (BM-MSC)conditioned media (CM) injection on uterine fibrosis after endometrial curettage in rabbit (Masson trichrome staining) A, The normal morphology of uteri was demonstrated in the intact group. B, In curettage group, uteri were with moderate abnormal morphology and thinner endometrium after 30 days. C, Treatment group after injection of BM-MSC-CM after 30 days. D, Treatment of group after injection of BM-MSC after 30 days (Scale bars $=200 \mu \mathrm{m})$. 


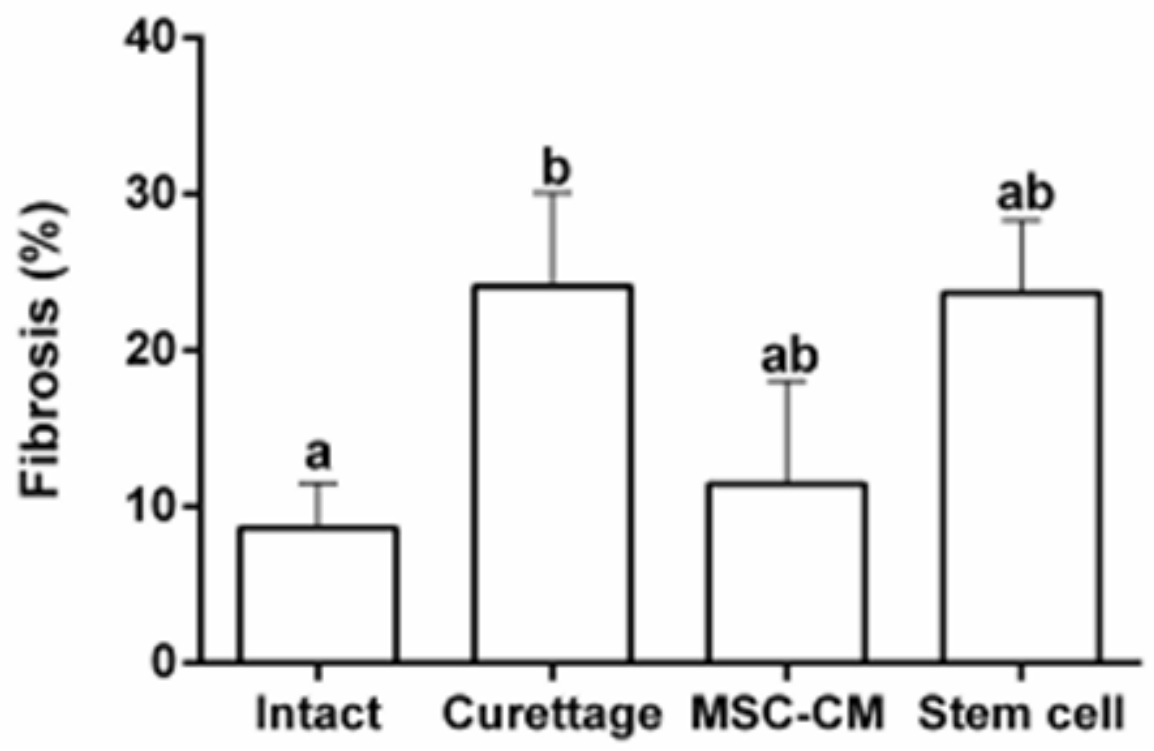

Figure 5

Mean and standard error of amount of collagen fibers in endometrial layer to compare the preventive effects of intrauterine bone marrow-derived stem cell (MSC)-conditioned media (CM) injection on uterine fibrosis after endometrial curettage in rabbit. a,b different superscript letters show statistical significant difference $(P<0.05)$. 

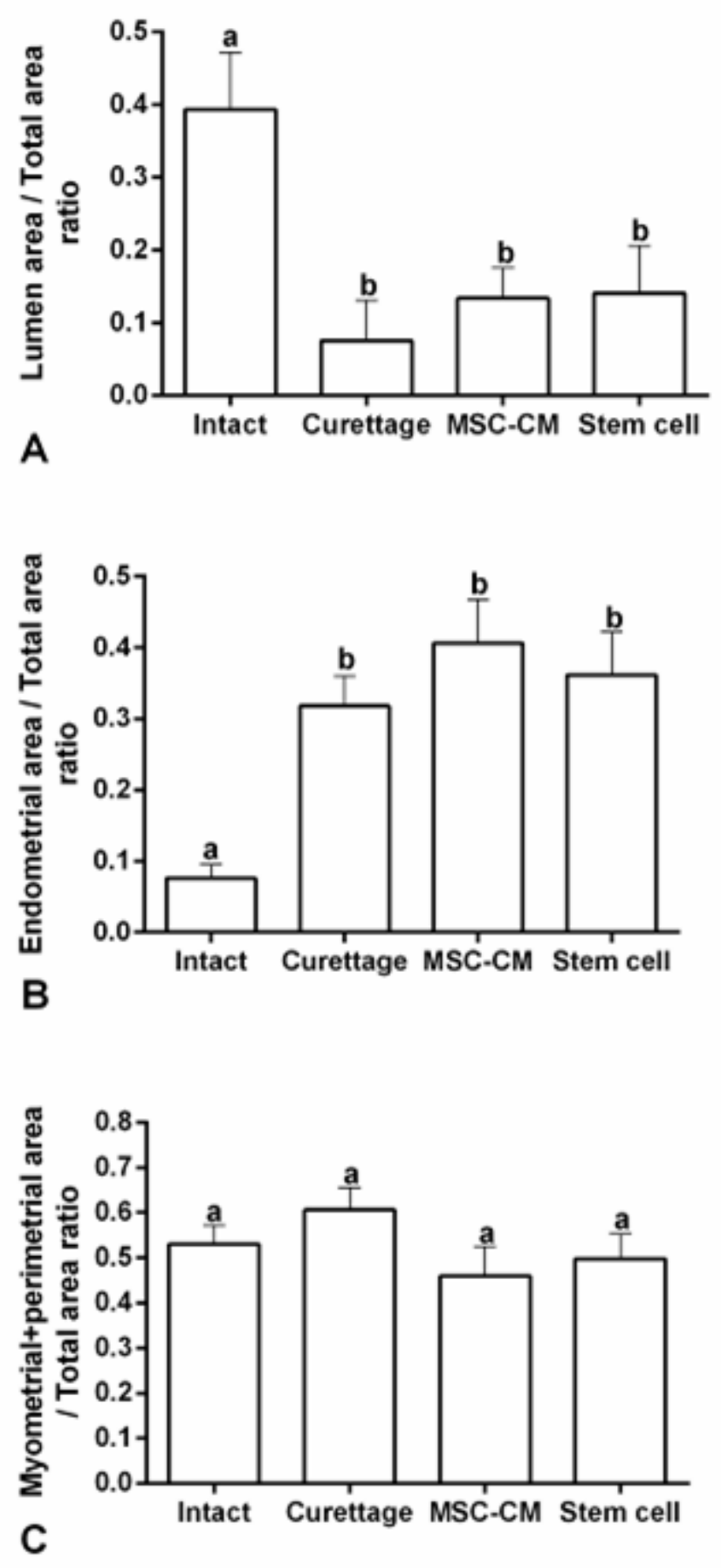

Figure 6

Mean and standard error of the uterine histomorphometric indices to compare the preventive effects of intrauterine bone marrow-derived stem cell (MSC)-conditioned media (CM) injection on uterine fibrosis after endometrial curettage in rabbit. A, Lumen area/total area ratio, B, Endometrial area/total area ratio, $\mathrm{C}$, Myometrial + perimetrial area/total area ratio. a,b different superscript letters show statistical significant difference $(P<0.05)$. 

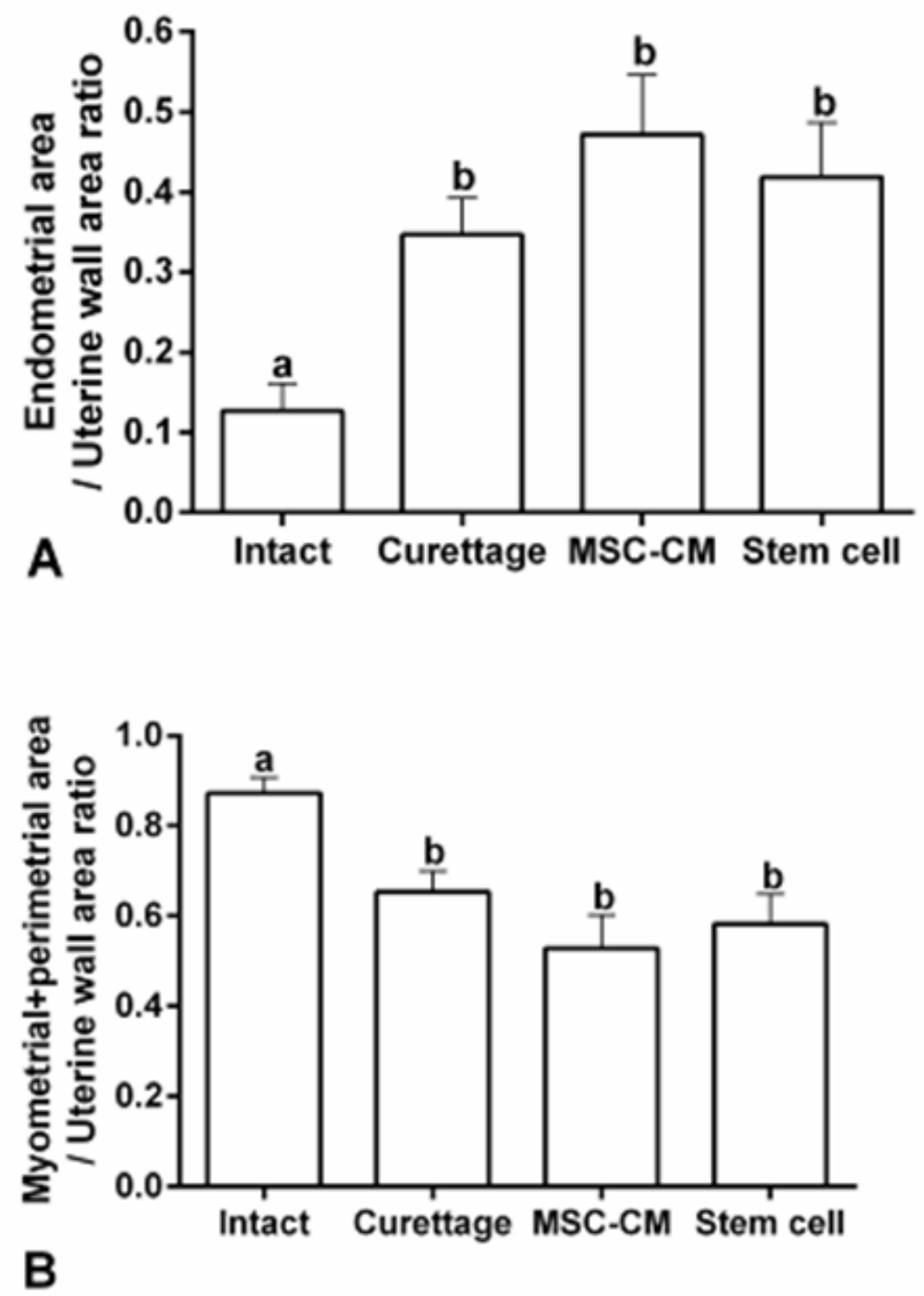

Figure 7

Mean and standard error of the uterine histomorphometric indices to compare the preventive effects of intrauterine bone marrow-derived stem cell (MSC)-conditioned media (CM) injection on uterine fibrosis after endometrial curettage in rabbit. A, Endometrial area/uterine wall area ratio, B, Myometrial + perimetrial area/uterine wall area ratio. a,b different superscript letters show statistical significant difference $(P<0.05)$. 